\title{
Risco cardiometabólico, estado nutricional e perfil lipídico em adolescentes do sexo masculino
}

\author{
Géssyca Corzino Medina, Dayane de Castro Morais, Silvia Eloiza Priore
}

\begin{abstract}
Resumo
A adolescência compreende a faixa etária dos 10 aos 19 anos, marcada por mudanças fisiológicas, psicossociais, comportamentais, culturais e emocionais. Avaliar o estado nutricional e para identificar possíveis riscos e agravos nutricionais é importante. Na avaliação do estado nutricional é interessante incluir a Relação Cintura Estatura (RCE), por esta ser considerada bom indicador para avaliação da obesidade e adiposidade central, e relacionar-se aos fatores de risco cardiovascular mais eficazmente do que o IMC isoladamente, sendo um método simples, barato e aplicável à população em geral. Avaliar o risco cardiometabólico, estado nutricional e perfil lipídico de adolescentes do sexo masculino. Participaram da pesquisa, adolescentes do sexo masculino do $1^{\circ}$ ano de um colégio de Viçosa, voluntários de um projeto de extensão no período de 2012 a 2015 . Os alunos foram convidados a realizar avaliação antropométrica e exames bioquímicos, mediante autorização dos pais ou responsáveis que assinaram o Termo de Consentimento Livre e Esclarecido (TCLE). Utilizou-se do índice de massa corporal (IMC), baseando-se nos pontos de corte e referencial antropométrico preconizados pela World Health Organization (2007), segundo sexo e idade. A categoria excesso de peso engloba os indivíduos com sobrepeso e obesidade. Para avaliação do risco cardiometabólico utilizou-se a relação cintura estatura (RCE), sendo valores de $\mathrm{RCE} \geq 0,5$, indicativas deste risco. Para os exames bioquímicos de perfil lipídico e triglicerídeos adotou-se pontos de corte da I Diretriz de Prevenção da Aterosclerose na Infância e Adolescência, 2005. Foi utilizado os softwares SPSS for Windows, versão 21.0 e STATA, versão 13.0, para analise dos dados. A magnitude das associações entre as variáveis realizadas utilizando Odds Ratio e Razão de Prevalência. Verificou-se a distribuição dos dados por meio de teste de Kolmogorov-Smirnov. Avaliou-se coeficiente de correlação de Spearman para verificar a relação entre as mesmas. Foram avaliados 73 adolescentes com idade entre 14 e 17 anos e mediana de idade de 15,1 anos. Dos quais 17,8\% ( $\mathrm{n}=13)$ apresentavam risco cardiometabólico pela RCE e excesso de peso, 34,2\% ( $\mathrm{n}=25)$ colesterol aumentado, 21,9\% $(\mathrm{n}=16)$ LDL alterado, 42,5\% $(\mathrm{n}=32)$ HDL abaixo do recomendado, 23,3\% $(n=17)$ com triglicerídeos alterados, 64,4\% $(n=47)$ apresentavam alguma alteração lipídica, 17,8\% (n=13) uma alteração metabólica e 8\% $(n=6)$ mais de uma alteração metabólica. Pode-se observar associação entre a RCE e presença de excesso de peso (RP=159,1; 20,263-1255,48), uma alteração metabólica $(\mathrm{RP}=25,38 ; 5,626-114,523)$ e mais de uma alteração metabólica $(\mathrm{RP}=10,08$; 3,297-30,812). A Relação Cintura Estatura correlacionou-se com o IMC/Idade ( $\mathrm{Z}$ escore) ( $\mathrm{r}=0,838$; $\mathrm{p}<0,001)$ e negativamente com HDL $(r=-0,254 ; \mathrm{p}<0,030)$. Conclui-se que a Relação Cintura Estatura é um bom índice para pressupor risco cardiometabólico em adolescentes, uma vez que associou-se e correlacionou-se com variáveis antropométricas e alterações nas frações lipídicas, além de ser um método simples e barato. Vale destacar a importância de utilizar métodos de avaliação nutricionais associados, já que os parâmetros bioquímicos isolados não apresentaram relação, porém quando associados ao estado nutricional, houve associação.
\end{abstract}

Descritores: Adolescentes; Risco Cardiometabólico; Estado Nutricional. 